\title{
Transformaciones $\alpha \leftrightarrow \beta$ en la aleación Ti-6Al-6V-2Sn: caracterización y características mecánicas y microestructurales
}

\author{
P. Tarín*, A. García-Simón*, N.M. Piris*, J.M. Badía* y J.M. Antoranz" \\ Resumen Para estudiar la cinética de la transformación $\alpha \leftrightarrow \beta$ en la aleación Ti-6Al-6V-2Sn, del tipo \\ $\alpha+\beta$, y valorar el porcentaje de fase $\alpha$ no trasformada ( $\alpha$ primaria) y la influencia en \\ transformaciones posteriores en enfriamiento, se realizaron ensayos de análisis térmico \\ (diferencial y dilatométrico), estudios metalográficos y medidas de dureza, tras tratamientos \\ térmicos controlados. Partiendo del estado de entrega (mill annealed), se realizaron \\ tratamientos de solución, mediante calentamiento de $1 \mathrm{~h}$ entre $650^{\circ} \mathrm{C}$ y $1.050^{\circ} \mathrm{C}$, con \\ enfriamiento en agua con agitación. Posteriormente, se han sometido las muestras a un \\ calentamiento a $570{ }^{\circ} \mathrm{C}$ durante $4 \mathrm{~h}$ y enfriamiento final al aire. Finalmente, se \\ determinaron las características en tracción.
}

Palabras clave Titanio. Tratamiento térmico. Transformación. Dilatometría. Análisis térmico diferencial. Propiedades mecánicas.

\section{$\alpha \leftrightarrow \beta$ transformation, mechanical properties and microestructural characterization of Ti-6Al-6V-2Sn alloy}

\begin{abstract}
In Ti-6Al-6V-2Sn alloy, $\alpha \leftrightarrow \beta$ transformation kinetics and other transformation characteristics, as well as its influence on subsequent cooling transformations, have been evaluated by differential and dilatometric thermal analisys, hardness measurements and metallographic observation, after performing controlled thermal treatments. Starting from the mill annealed condition, samples were heated at temperatures between $650-1050^{\circ} \mathrm{C}$ for $1 \mathrm{~h}$, then water quenched and subsequently heated at $570^{\circ} \mathrm{C}$ for $4 \mathrm{~h}$, air cooled. Finally, the mechanical properties of samples heat treated as indicated above have been obtained.
\end{abstract}

Keywords Titanium. Heat treating. Transformation. Differential thermal analisys. Dilatometry. Mechanical properties.

\section{INTRODUCCIÓN}

La aleación Ti-6Al-6V-2Sn puede, al igual que otras aleaciones del tipo $\alpha+\beta$, ser sometida a tratamientos de solución. En dichos tratamientos, la fase $\alpha$, al calentarse, se va transformando progresivamente en fase $\beta$ hasta alcanzar la temperatura $\beta$-transus, en la que se completa dicha reacción. Para poder llevar a cabo tratamientos de solución en los que se deja sin transformar un determinado porcentaje de fase $\alpha$ (primaria), es necesario identificar y cuantificar la cinética de esa transformación. Para esta aleación, se ha determinado una curva experimental que proporciona el porcentaje de fase $\alpha$ primaria en función de la temperatura de solución, similar a la ya obtenida en estudios previos para la aleación Ti-6-4 ${ }^{[1]}$. Para obtener dicha curva se ha empleado el tradicional método metalográfico y valoraciones realizadas en las curvas obtenidas en ensayos de análisis térmico diferencial y dilatométrico.

Se han realizado medidas de la dureza y ensayos de tracción de probetas sometidas a tratamientos de solución a temperaturas crecientes y maduración posterior. En esas mismas probetas, se midió la conductividad eléctrica, resultando valores del orden de $0,57 \pm 0,01 \mathrm{MS} / \mathrm{m}$, tras tratamientos de solución (y $0,60 \pm 0,01 \mathrm{MS} / \mathrm{m}$, tras maduración) con variaciones con la temperatura de tratamiento, pequeñas y poco representativas. 


\section{ENSAYOS REALIZADOS}

\subsection{Material y tratamientos térmicos}

Se partió de una barra de aleación Ti-6Al-6V-2Sn de $60 \mathrm{~mm}$ de diámetro, en estado MA (mill annealed). Muestras de la misma se calentaron durante $1 \mathrm{~h}$, en vacío, a temperaturas entre $650^{\circ} \mathrm{C}$ y $1.050^{\circ} \mathrm{C}$, con enfriamiento en agua agitada. La maduración posterior fue de $4 \mathrm{~h}$ a $570^{\circ} \mathrm{C}$, enfriando en aire.

\subsection{Análisis térmico diferencial y dilatométrico}

Los ensayos de ATD y dilatométricos, se realizaron con calentamientos y enfriamientos a $10^{\circ} \mathrm{C} / \mathrm{min}$.

\subsection{Ensayos mecánicos}

Los ensayos de tracción se realizaron de acuerdo con la norma EN 10002-1, sobre probetas cilíndricas de $8 \mathrm{~mm}$ de diámetro y midiendo deformaciones en una longitud-base de $50 \mathrm{~mm}$.

\section{RESULTADOS}

\subsection{Estudio metalográfico}

Las probetas se pulieron electrolíticamente $\mathrm{e}^{[2]}$ y se observaron sin ataque. La microestructura inicial en una sección longitudinal de la barra presentaba granos muy alargados de fase $\alpha$ en una proporción que se valoró, así como la cantidad de fase $\alpha$ primaria o no transformada en cada calentamiento. La evolución de la microestructura con la temperatura de solución es similar a la obtenida en otras aleaciones del tipo $\alpha+\beta$, como la Ti-6Al-4V ${ }^{[1]}$.

En la figura 1 se muestran las microestructuras tras dos tratamientos de solución. Tras la solución a $850^{\circ} \mathrm{C}$, todavía puede apreciarse la dirección de forja. Tras solución a $950^{\circ} \mathrm{C}$, el grano de $\beta$ obtenido en calentamiento ha crecido y se ha transformado en martensita en el enfriamiento; y la pequeña cantidad de fase $\alpha$ primaria está inhibiendo el crecimiento de grano de la fase $\beta$. Tras la solución a $975^{\circ} \mathrm{C}$, por haber rebasado la temperatura de $\beta$-transus, no queda fase $\alpha$ primaria y la martensita se presenta en granos muy bastos $(\approx 0,5 \mathrm{~mm})$ heredados de la fase $\beta$.

\subsection{Análisis térmico diferencial}

En la figura 2 se muestra el termograma obtenido en un segundo calentamiento, con resultados simi-
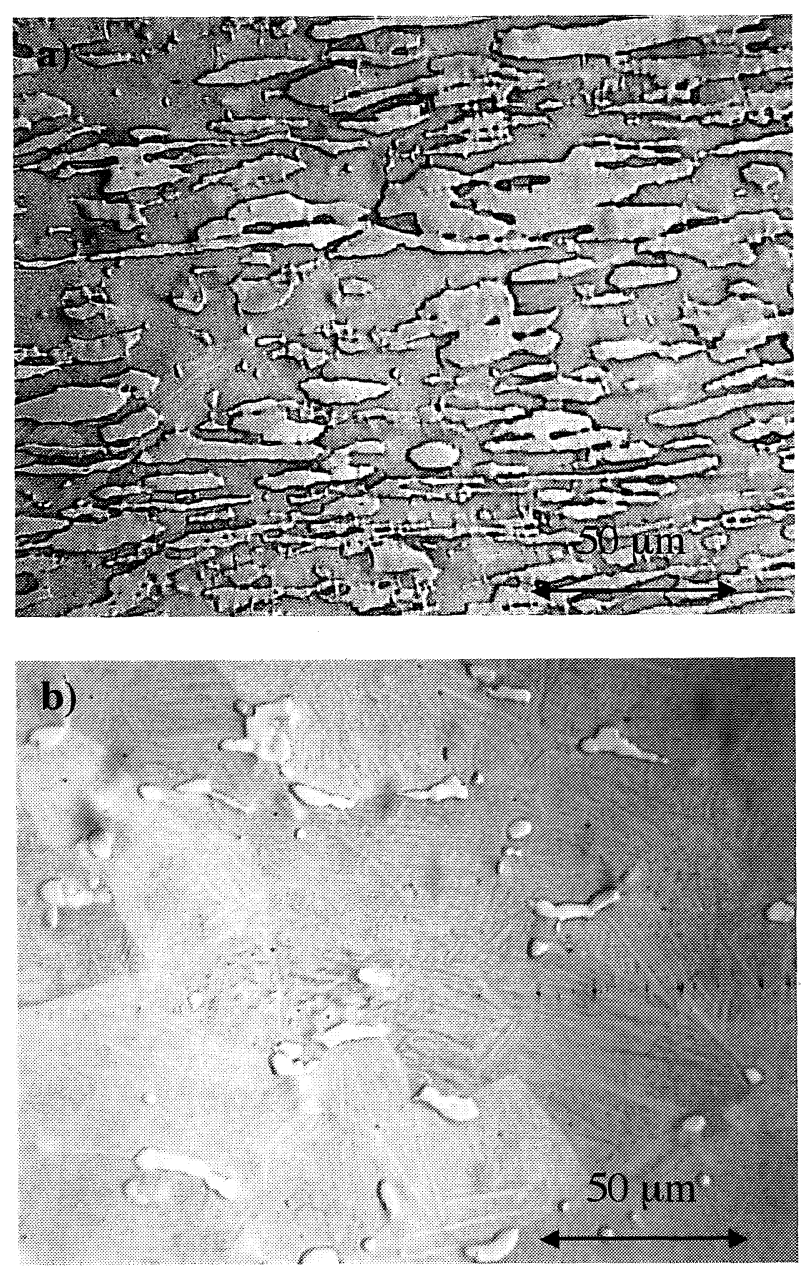

Figura 1. Microestructuras. a) Tras solución a $850{ }^{\circ} \mathrm{C}$; b) Tras solución a $950^{\circ} \mathrm{C}$.

Figure 1. Microstructures. a) After $850^{\circ} \mathrm{C}$ solution treatment. b) Affer $950^{\circ} \mathrm{C} \mathrm{ST}$.

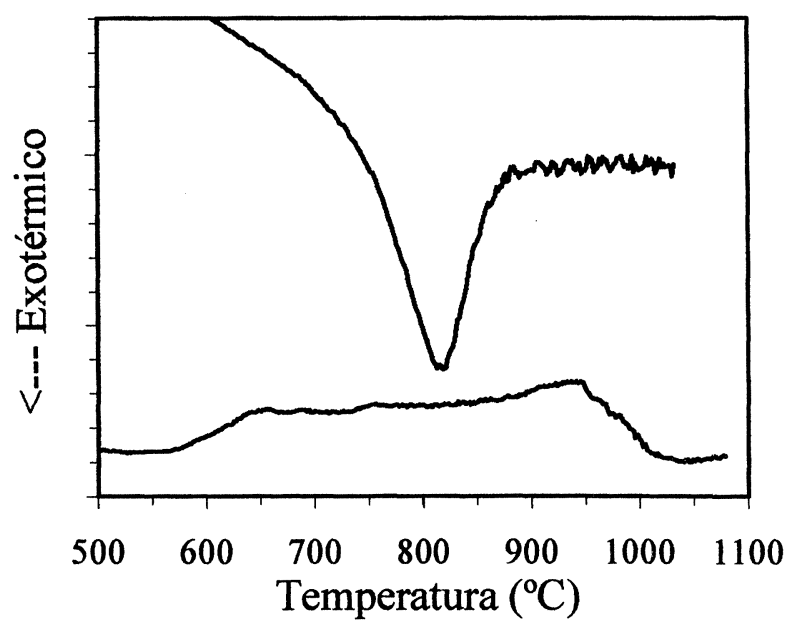

Figura 2. Análisis térmico diferencial.

Figure 2. Differential thermal analisys.

lares a los de la aleación Ti-6Al-4V ${ }^{[1]}$. En el gráfico correspondiente a un primer calentamiento, se 
aprecia, además, en el margen de $725-800^{\circ} \mathrm{C}$, un ligero proceso exotérmico que no se repite en calentamientos siguientes, por lo que puede corresponder al proceso de recristalización. La transformación $\alpha \rightarrow \beta$ se detecta como un proceso endotérmico con inicio no muy bien definido y un final a temperatura inferior a los $1.000^{\circ} \mathrm{C}$. En enfriamiento se aprecia bien la reacción exotérmica correspondiente a la transformación $\beta \rightarrow \alpha$.

\subsection{Análisis dilatométrico}

La figura 3 recoge el resultado de dos ensayos dilatométricos. En la figura 3a), obtenida con probeta de eje transversal a la barra, la transformación $\alpha \rightarrow \beta$ se identifica como una disminución apreciable de volumen, como en trabajos similares ${ }^{[1,3}$ y 4$]$, y tiene lugar entre los 800 y los $950^{\circ} \mathrm{C}$.
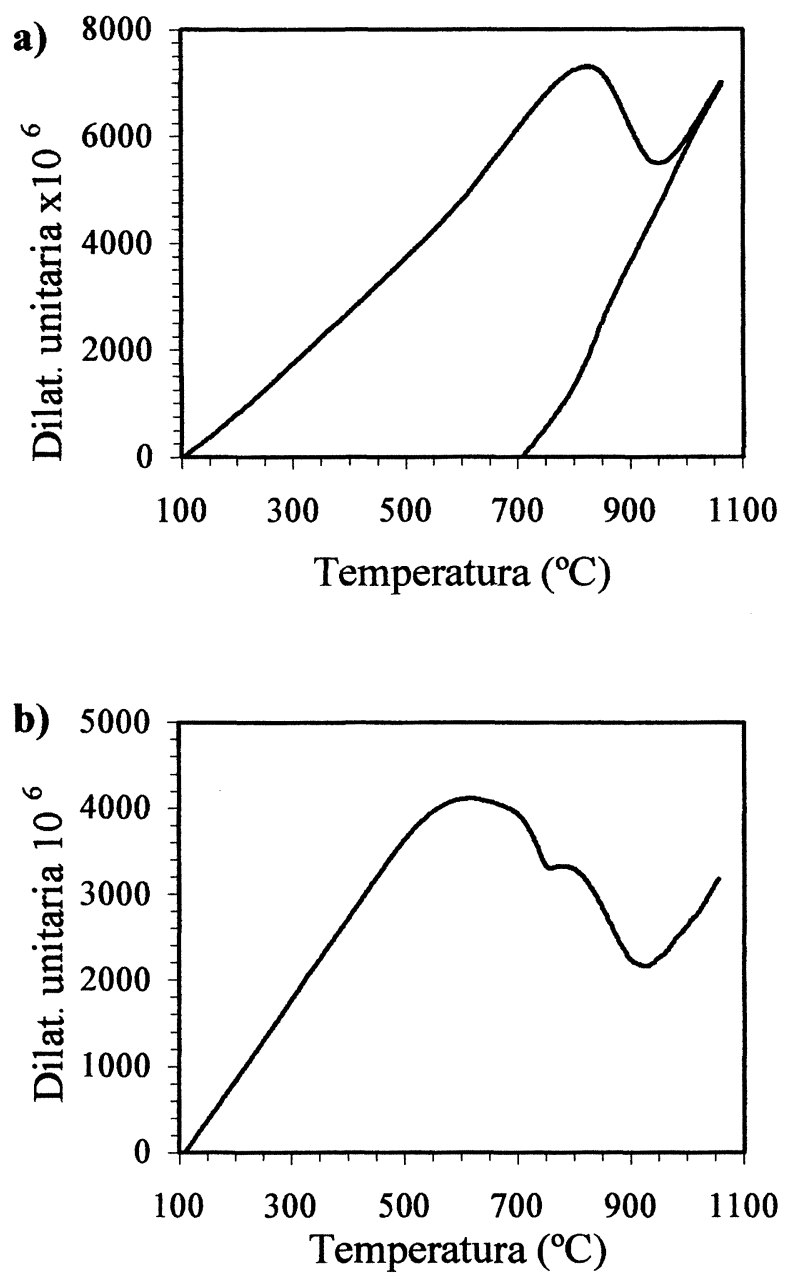

Figura 3. Análisis térmico dilatométrico. a) probeta transversal); b) probeta longitudinal.

Figure 3. Dilatometric thermal analisys. a) short direction); b) long direction.
En el margen de los $500-600{ }^{\circ} \mathrm{C}$ se aprecia un progresivo aumento de volumen que corresponde a una discontinuidad en el coeficiente de dilatación ${ }^{[5]}$. En el enfriamiento el cambio de volumen es, cuantitativamente, menor.

La disminución de volumen en la transformación tiene relación con ésta y con la deformación por fluencia a temperaturas elevadas, debido al peso del transductor sobre la probeta, lo que obliga a considerar el ensayo como un ensayo termomecánico ${ }^{[1]}$. Ello complica, a priori, la interpretación de la gráfica pero, dado que el proceso de fluencia está activado por la difusión que acompaña a la propia transformación, las conclusiones y valoraciones de la transformación son, normalmente, suficientemente válidas.

En calentamientos siguientes, en las mismas condiciones que el primero, la transformación se produce, prácticamente, en el mismo margen de temperaturas, aunque con diferencias en el cambio de volumen asociado a la transformación, debido, por un lado, a consecuencias del pequeño cambio de volumen real asociado a ella y a la tendencia a textura de estas aleaciones y, por otro, a que la resistencia a fluencia es mucho mayor debido al grano muy basto originado en el primer calentamiento hasta una temperatura superior a la del $\beta$-transus.

La figura $3 \mathrm{~b}$ ) muestra el resultado obtenido en un primer calentamiento de una probeta longitudinal próxima a la superficie de la barra. El resultado resulta más difícil de interpretar desde el punto de vista de la transformación $\alpha \rightarrow \beta$, ya que presenta una acusada anomalía en el margen de los 650 . $800{ }^{\circ} \mathrm{C}$, que puede asociarse al proceso de recristalización y la fluencia activada por la difusión que acompaña a ese proceso. Las temperaturas coinciden con las anotadas como posibles para ese proceso, deducidas de ensayos de ATD. Otros ensayos con probetas equivalentes no dieron los mismos resultados lo que, si bien puede explicarse por razones ya apuntadas antes a las que debe añadirse la gran tendencia a segregación de estas aleaciones, significa que los resultados de algunos ensayos pueden inducir a errores en su interpretación.

\subsection{Valoración de la transformación $\alpha \leftrightarrow \beta$}

En la figura 4a) se muestra el porcentaje de fase $\alpha$ primaria no transformada en función de la temperatura de solución, obtenido por distintos métodos, pudiendo deducirse un buen acuerdo entre los resultados de ellos. La figura $4 \mathrm{~b}$ ) muestra los 

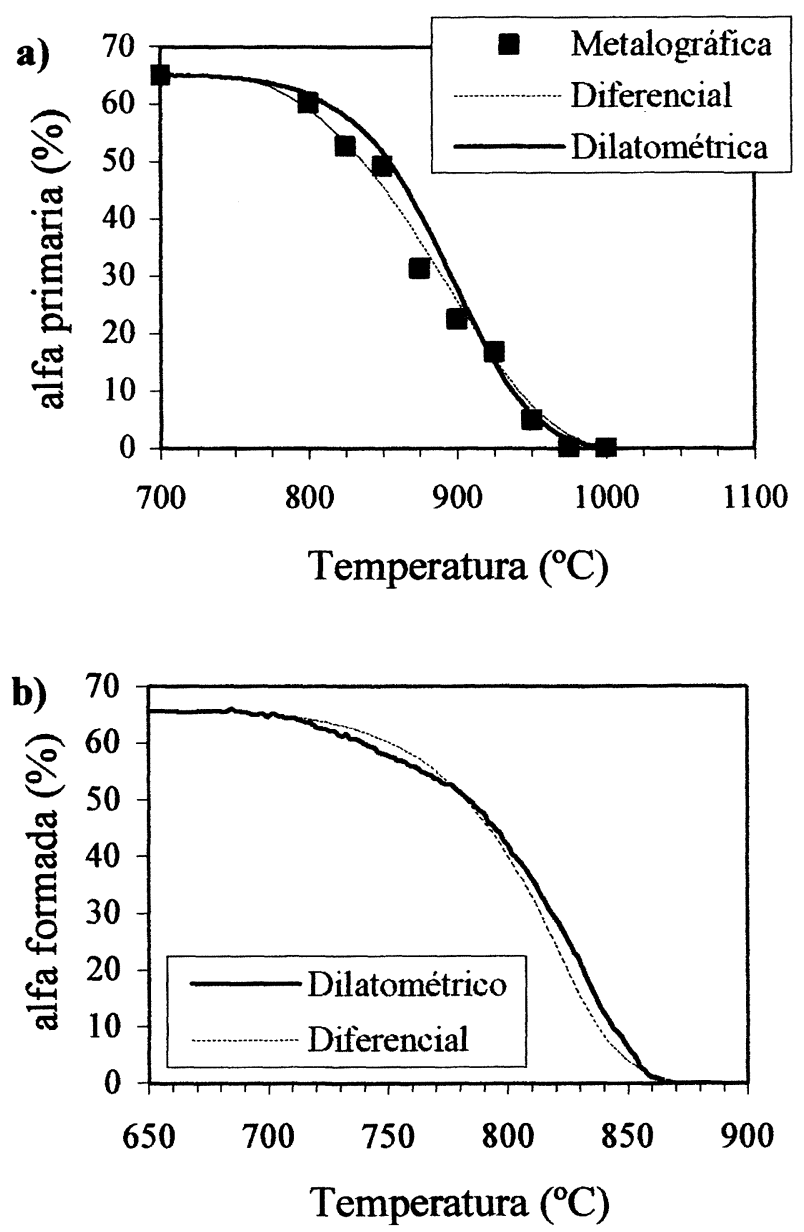

Figura 4. Valoración de la transformación $\alpha \leftrightarrow \beta$ por diversos métodos. a) en calentamiento a $10^{\circ} \mathrm{C} / \mathrm{min}$.; b) en enfriamiento a $10^{\circ} \mathrm{C} / \mathrm{min}$.

Figure 4. $\alpha \leftrightarrow \beta$ transformation valuation, by different methods. a) heating, $10^{\circ} \mathrm{C} / \mathrm{min}$; b) cooling, $10^{\circ} \mathrm{C} / \mathrm{min}$.

resultados de la valoración de la transformación $\beta \rightarrow \alpha$ en enfriamiento, con un buen acuerdo entre los dos resultados. Otros autores han mostrado un buen acuerdo entre los resultados de estas técnicas y los de ensayos de medidas de conductividad eléctrica $^{[6]}$.

\subsection{Características mecánicas}

La figura 5 presenta los resultados de las medidas de dureza tras los tratamientos. Se comprueba cómo, a bajas temperaturas (hasta unos $800^{\circ} \mathrm{C}$ ), la dureza obtenida tras solución va disminuyendo ligeramente. Ello puede asociarse con el proceso de recristalización detectada en esas temperaturas por otras técnicas de ensayo ya citadas.

Al seguir aumentando la temperatura de solución, la dureza va siendo mayor, debido a la martensita o fase $\beta$, formadas en el temple. Ya no au-
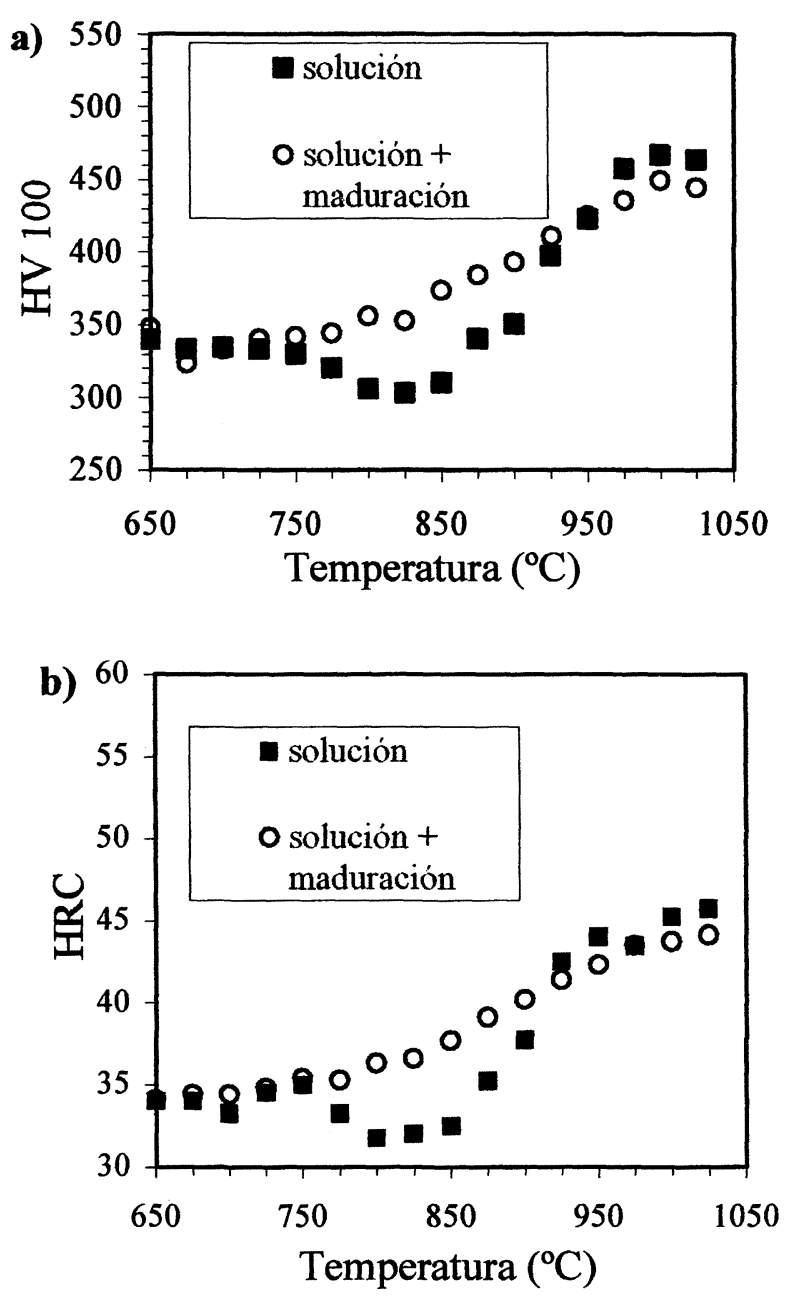

Figura 5. Variación de dureza con la temperatura de solución.

Figure 5. Hardness vs. solution temperature.

menta al alcanzar la correspondiente a la de $\beta$-transus.

La maduración origina un aumento de dureza en las probetas, con solución a bajas temperaturas, y una disminución en las de temperaturas altas. Ello, es debido a que la fase $\beta$ formada a temperaturas más bajas es más rica en elementos de aleación y origina en el temple una mayor proporción de $\beta$ metaestable (retenida) que aumenta su dureza en la maduración. Las de más altas temperaturas originan en el temple una mayor proporción de martensita que puede disminuir su dureza en la maduración. Los resultados son acordes con los resultados obtenidos en la aleación Ti-6Al-4V [1] $\mathrm{y}$, aunque las variaciones son cuantitativamente mayores, no son lo suficientemente significativas como para utilizar de modo fiable esas medidas como control del tratamiento de solución.

La figura 6 muestra los resultados de ensayos de tracción obtenidos al variar la temperatura de 

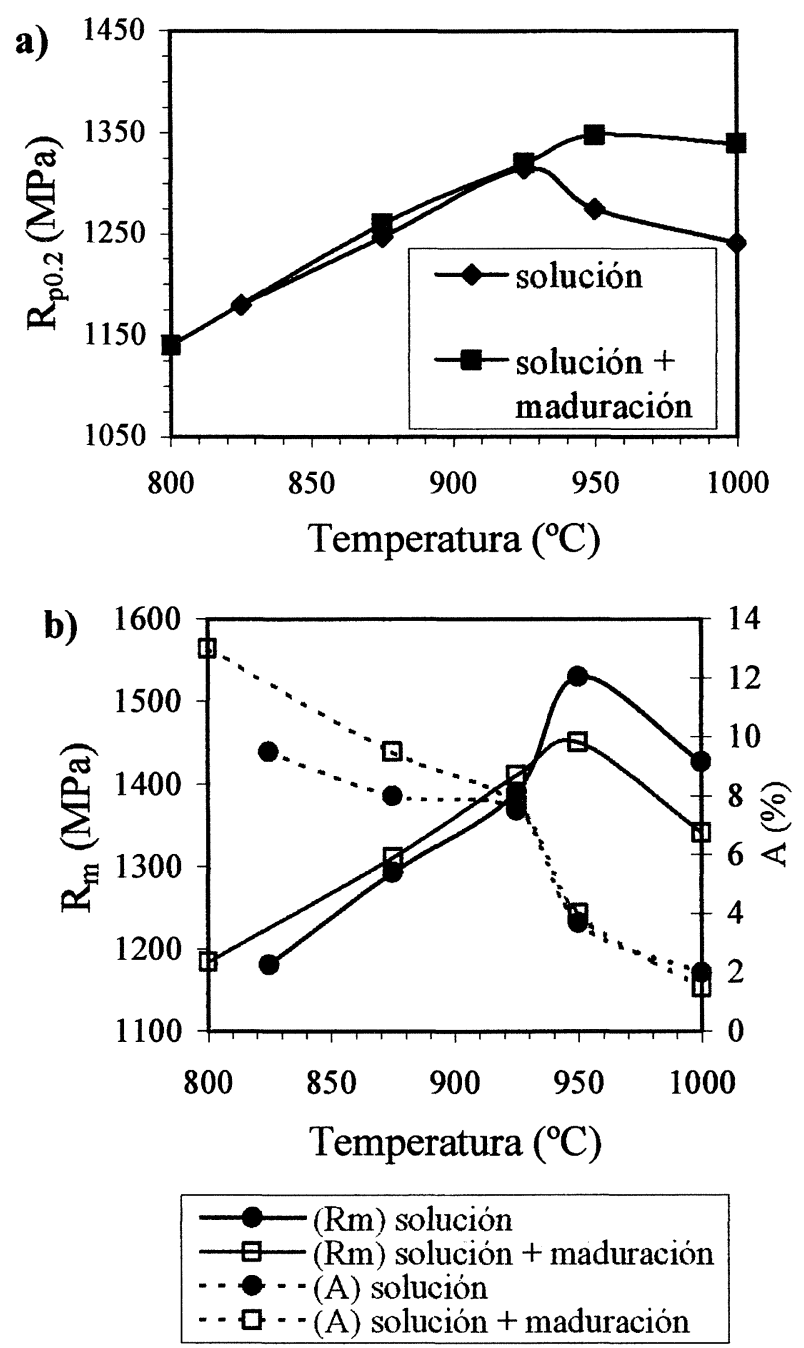

Figura 6. Variación de propiedades mecánicas en función de la temperatura de solución.

Figure 6. Mechanical properties vs. solution temperature.

solución. Las propiedades resistivas aumentan casi linealmente (y la plasticidad disminuye) con la temperatura de solución hasta los $925^{\circ} \mathrm{C}$ y, de modo muy diferente (incluso anómalo) para temperaturas más altas, para las que la plasticidad disminuye de forma muy acusada. Ello, es debido al gran aumento de tamaño de grano que se produce a temperaturas muy próximas (y superiores) a la temperatura de $\beta$-transus. Estos resultados, y los de dureza son, cualitativamente, similares a $\operatorname{otros}^{[7]}$.

De esos resultados puede deducirse que la temperatura de solución para obtener un comportamiento mecánico óptimo es una próxima, pero in- ferior, a los $925^{\circ} \mathrm{C}$ (quedando sin transformar $\approx 20$ $\%$ de $\alpha$ primaria), algo superior a la de $885^{\circ} \mathrm{C}$, recomendada por otros autores ${ }^{[5]}$ (a la que quedaría $\sin$ transformar $\approx 30 \%$ de $\alpha$ primaria).

\section{CONCLUSIONES}

El método más válido y representativo para valorar la transformación $\alpha \rightarrow \beta$ es el metalográfico. No obstante, los ensayos de ATD y dilatométricos, son un método rápido de valoración, con resultados de la evolución de la transformación válidos, a pesar de la dificultad de su interpretación y las variables ajenas a la propia transformación (recristalizaciones, fluencia y textura, segregaciones, etc.) que influyen en los resultados, sobre todo en la dilatometría.

De los resultados de los ensayos mecánicos se deduce que la temperatura de solución para obtener un comportamiento mecánico óptimo es la próxima a los $925^{\circ} \mathrm{C}$, por alcanzarse valores resistivos casi máximos, sin pérdida muy acusada de plasticidad. A esa temperatura queda sin transformar $\approx 20 \%$ de $\alpha$ primaria.

Las medidas de dureza ponen, sólo relativamente, de manifiesto las transformaciones que se producen en la solución y en là maduración, por lo que no constituyen un método muy fiable para la valoración o control de la transformación.

\section{REFERENCIAS}

[1] P. Tarín, A.G. Simón, N.M. Piris, J.M. Badía y J.M. Antoranz, Bol. Soc. Esp. Ceram. Vidrio 43 (2004) 267-272.

[2] I. Fernández, L. Rubio, J.M. Antoranz, J.M. Badía, P. TARín y A. García, VII Congreso Nacional de Tratamientos Térmicos y de Superficie, Madrid, 1998.

[3] F. Le Maître, Mem. Sci. Rev. Metall. LXVII (1970) 563. 574.

[4] P. TARÍn e I. GoÑI, V Asamblea General, CENIM, Madrid, 1981.

[5] ASM International, Materials Properties Data Handbook: Titanium alloys, 1994.

[6] S. BEIN y J. BECHET, Titanium '95: Science and Technology III, Birmingham (UK), 1995, pp. 2.353-2.360.

[7] Aerospace Structural Metals Handbook, Mechanical Properties Data Center, Department of Defense, SINDAS/USAF, Purdue University, USA, 2002. 